\title{
Sky localization of space-based gravitational wave detectors
}

\author{
Chao Zhang (张超), ${ }^{1, *}$ Yungui Gong (龚云贵) ${ }^{1, \dagger}$ Hang Liu (刘 \\ 航), ${ }^{2} \ddagger$ Bin Wang (王斌) $, 3,2, \S$ and Chunyu Zhang (张春雨) ${ }^{1,}$ 『 \\ ${ }^{1}$ School of Physics, Huazhong University of Science and Technology, Wuhan, Hubei 430074, China \\ ${ }^{2}$ School of Aeronautics and Astronautics, Shanghai Jiao Tong University, Shanghai 200240, China \\ ${ }^{3}$ Center for Gravitation and Cosmology, Yangzhou University, Yangzhou 225009, China.
}

\begin{abstract}
Localizing the sky position of the gravitational wave source is a key scientific goal for gravitational wave observations. Employing the Fisher information matrix approximation, we compute the angular resolutions of LISA and TianQin, two planned space-based gravitational wave detectors and examine how detectors' configuration properties, such as the orientation change of the detector plane, heliocentric or geocentric motion and the arm length etc., affect the accuracy of source localization for monochromatic sources at selected values of frequencies. We find that the amplitude modulation due to the annual changing orientation of the detector plane helps LISA get better accuracy in the sky localization and better sky coverage at frequencies below several $\mathrm{mHz}$, and its effect on TianQin is negligible although the orientation of TianQin's detector plane is fixed. At frequencies above roughly $30 \mathrm{mHz}$, TianQin's ability in the sky localization is better than LISA. Further we explore potential space detector networks for fast and accurate localization of the gravitational wave sources. The LISA-TianQin network has better ability in sky localization for sources with frequencies in the range $1-100 \mathrm{mHz}$ and the network has larger sky coverage for the angular resolution than the individual detector.
\end{abstract}

\section{INTRODUCTION}

Since the first gravitational wave (GW) event GW150914 observed by the Laser Interferometer Gravitational-Wave Observatory (LIGO) Scientific Collaboration and the Virgo Collaboration [1, 2], there have been tens of confirmed GW detections by ground-based GW observatories [1-13]. The ground-based GW observatories can measure GWs within frequency range $10-10^{3} \mathrm{~Hz}$ only, and are difficult to explore lower frequency band where a wealth of astrophysical signals reside. The proposed space-based observatories including LISA [14, 15], TianQin [16] and Taiji [17] are expected to detect GWs in the low-frequency regime.

Accurately localizing GW sources is important, since the source position is correlated with the physical properties of the binary star system which are necessary to understand the formation and evolution of the binary. Moreover, the accurate GW source localization may provide important information about the environments where such relativistic objects reside. In particular, the accurate knowledge of the GW source position is essential for the follow-up observations of counterparts and the statistical identification of the host galaxy if no counterpart is found, so that we can use GWs as standard sirens to explore the universe's expansion history and understand the problem of Hubble tension [18-22].

One ground-based GW observatory cannot localize GW source, since it is sensible to GW signals from nearly

\footnotetext{
* chao_zhang@hust.edu.cn

† Corresponding author. yggong@hust.edu.cn

¥ hangliu@sjtu.edu.cn

$\S$ wang_b@sjtu.edu.cn

I chunyuzhang@hust.edu.cn
}

all directions within a few seconds to minutes. It requires three or more ground-based GW detectors at widely separated sites to locate GW sources with the method of timing triangulation approximation [23-25]. However, space-based GW detector can measure GWs for months to years, the periodic Doppler shift due to the detector motion in space results in amplitude and phase modulations of the detected GW signals which encode information about the detector position and the angular position of the source. Therefore, a single space-based GW detector is able to locate the source position.

Like LISA, Taiji is composed of a triangle of three spacecrafts with a larger separation distance in a heliocentric orbit ahead instead of behind the Earth by about $20^{\circ}$ [17]. The similarities in configurations construe that similar to LISA, Taiji's angular resolution depends similarly on the type of signal and on how much other information must be extracted. Similarities and complements between LISA and Taiji imply that LISA-Taiji network can effectively help to accurately localize GW sources, since the angular resolution measurements for the network depend on the configuration angle and separation of the two constellations [26, 27]. The network was estimated to improve the angular resolution over 10 times than each individual LISA or Taiji detector [28].

Unlike LISA and Taiji, TianQin has a geocentric orbit configuration with three spacecrafts orbiting the Earth and further rotating around the Sun together with the Earth. The normal vector of TianQin's detector plane points to the source RX J0806.3+1527 at $\left(\theta_{t q}=-4.7^{\circ}, \phi_{t q}=120.5^{\circ}\right)$. TianQin is slightly more sensitive to GWs with higher frequency than LISA. The precision of the parameter estimation and the sky localization of equal mass supermassive black hole binary systems with masses in the range $10^{5}-10^{7} M_{\odot}$ for TianQin was discussed in [29]. It was further argued that the 
LISA-TianQin network can improve the sky localization of Galactic double white dwarf binaries up to 3 orders of magnitude [30], if compared with single TianQin observation.

For TianQin, considering its special configuration and design, it is important to fully study its angular resolution and uncover its dependencies. It is needed to be clarified in TianQin project how information about the source position encodes in Doppler shift, the translational motion of the detector relative to the source, detector's changing orientation, the rotation period of spacecrafts and the arm length of the detector. Different from LISA, whose amplitude modulation improves the sky localization accuracy below $1 \mathrm{mHz}[31,32]$, the detector plane of TianQin points to a fixed direction so there is no amplitude modulation in TianQin which is more sensitive to signals above several $\mathrm{mHz}$. It is interesting to explore how much the sky localization accuracy of TianQin is affected in the absence of the amplitude modulation. On the other hand, the Doppler modulation becomes stronger as the frequency of GWs increases and it has the equatorial pattern [31-33], it is natural to ask how the Doppler modulation affects the sky localization accuracy of TianQin and how to compare it with other space-based detectors at frequencies above $10 \mathrm{mHz}$. Moreover, there are other questions to be answered, for example: How the equatorial pattern affects the sky localization accuracy? Is it possible that TianQin has better sky localization accuracy than LISA and Taiji even if it has shorter arm length? Neglecting differences in orbit configurations, if we explore the potential TianQin-LISA network, can it perform better in source localization than TaijiLISA network? We will carefully discuss these issues and present sky maps of angular resolutions for monochromatic sources to identify the sensitive regions of different detectors. These discussions are helpful to improve the detector design. Throughout the work, we will employ the Fisher information matrix approximation (FIM) to give robust estimation of the sky localization of the source with a high signal to noise ratio (SNR) where the inverse of FIM gives the covariance matrix of the parameters.
The organization of the paper is as follows. In Sec. II, we review the FIM method of signal analysis. In Sec. III, we devise several fiducial detectors to discuss the effects of different factors on the sky localization. The orbits for these detectors are presented in the Appendix. Then in Sec. IV we use these results to analyze the angular resolutions of TianQin, compare the result with LISA and finally explore the combined LISA-TianQin network. We present our conclusions and discussions in the last section.

\section{SIGNAL ANALYSIS}

For a GW signal

$$
h_{i j}(t)=\sum_{A} e_{i j}^{A} h_{A}(t)
$$

the output in the detector $\alpha$ is

$$
H_{\alpha}(t)=\sum_{A} F_{\alpha}^{A} h_{A}(t)+\hat{n}_{\alpha}(t)
$$

where $A=+, \times$ stands for the plus and cross polarizations, $e_{i j}^{A}$ is the polarization tensor, $\hat{n}_{\alpha}(t)$ is the detector noise, the angular response function $F_{\alpha}^{A}$ for the polarization $A$ is

$$
F_{\alpha}^{A}=\sum_{i, j} D_{\alpha}^{i j} e_{i j}^{A}
$$

and $D_{\alpha}^{i j}$ is the detector tensor. In GR, there are two polarizations. For alternative theory of gravity, there may exist up to six polarizations [34-42]. In this paper, we consider GR only. For equal arm space-based interferometric detector with a single round trip light travel, the detector tensor for a monochromatic GW with the frequency $f$ propagating along the direction $\hat{\omega}$ is

$$
D_{\alpha}^{i j}=\frac{1}{2}\left[\hat{u}_{\alpha}^{i} \hat{u}_{\alpha}^{j} T\left(f, \hat{u}_{\alpha} \cdot \hat{\omega}\right)-\hat{v}_{\alpha}^{i} \hat{v}_{\alpha}^{j} T\left(f, \hat{v}_{\alpha} \cdot \hat{\omega}\right)\right]
$$

where $\hat{u}_{\alpha}$ and $\hat{v}_{\alpha}$ are the unit vectors along the arms of the detector $\alpha, T\left(f, \hat{u}_{\alpha} \cdot \hat{\omega}\right)$ is $[43,44]$

$$
T\left(f, \hat{u}_{\alpha} \cdot \hat{w}\right)=\frac{1}{2}\left\{\operatorname{sinc}\left[\frac{f}{2 f^{*}}\left(1-\hat{u}_{\alpha} \cdot \hat{\omega}\right)\right] \exp \left[-i \frac{f}{2 f^{*}}\left(3+\hat{u}_{\alpha} \cdot \hat{\omega}\right)\right]+\operatorname{sinc}\left[\frac{f}{2 f^{*}}\left(1+\hat{u}_{\alpha} \cdot \hat{\omega}\right)\right] \exp \left[-i \frac{f}{2 f^{*}}\left(1+\hat{u}_{\alpha} \cdot \hat{\omega}\right)\right]\right\}
$$

$\operatorname{sinc}(x)=\sin x / x, f^{*}=c /(2 \pi L)$ is the transfer frequency of the detector, $c$ is the speed of light and $L$ is the arm length of the detector. With the signal $H_{\alpha}$, we define SNR as

$$
\begin{aligned}
\rho^{2} & =\sum_{\alpha}\left(H_{\alpha} \mid H_{\alpha}\right) \\
& =4 \sum_{\alpha} \int_{0}^{\infty} d f \frac{1}{S_{n, \alpha}(f)} H_{\alpha}(f) H_{\alpha}^{*}(f),
\end{aligned}
$$

where the power spectral density $S_{n, \alpha}(f)$ satisfies $\left\langle\tilde{n}_{\alpha}(f) \tilde{n}_{\alpha}^{*}(f)\right\rangle=\frac{1}{2} \delta\left(f-f^{\prime}\right) S_{n, \alpha}$. For space-based interferometers, the noise power spectral density $S_{n}(f)$ is $[16,45]$

$$
S_{n}(f)=\frac{S_{x}}{L^{2}}+\frac{4 S_{a}}{(2 \pi f)^{4} L^{2}}\left(1+\frac{10^{-4} \mathrm{~Hz}}{f}\right) .
$$

For TianQin, the acceleration noise is $\sqrt{S_{a}}=$ $10^{-15} \mathrm{~m} \mathrm{~s}^{-2} / \mathrm{Hz}^{1 / 2}$, the displacement noise is $\sqrt{S_{x}}=$ 


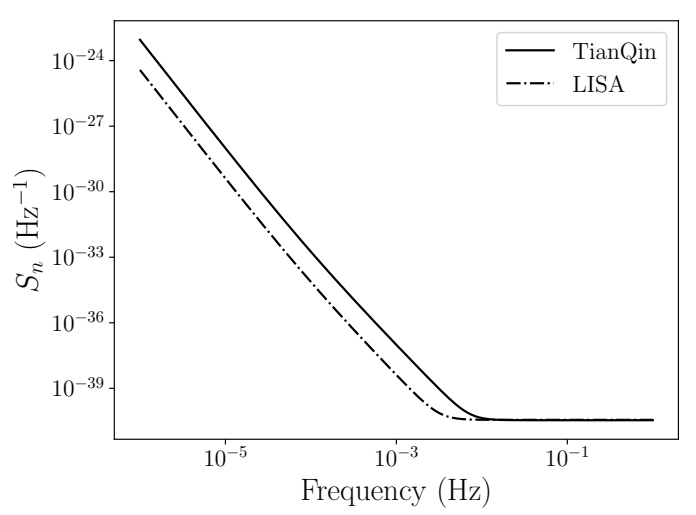

FIG. 1. The noise curves for LISA and TianQin.

$1 \mathrm{pm} / \mathrm{Hz}^{1 / 2}$ and the arm length is $L_{t}=1.7 \times 10^{5} \mathrm{~km}$ [16]. Its transfer frequency is $f_{t}^{*}=0.28 \mathrm{~Hz}$.

For LISA, the acceleration noise is $\sqrt{S_{a}}=3 \times$ $10^{-15} \mathrm{~m} \mathrm{~s}^{-2} / \mathrm{Hz}^{1 / 2}$, the displacement noise is $\sqrt{S_{x}}=$ $15 \mathrm{pm} / \mathrm{Hz}^{1 / 2}$ and the arm length is $L_{s}=2.5 \times 10^{6} \mathrm{~km}$ [15]. Its transfer frequency is $f_{s}^{*}=0.02 \mathrm{~Hz}$. The noise curves for LISA and TianQin are shown in Fig. 1. The ratios of the noise $S_{n}\left(f_{0}\right)$ between LISA and TianQin are 23 at $f_{0}=1 \mathrm{mHz}, 1.2$ at $f_{0}=10 \mathrm{mHz}$ and 0.9 at $f_{0}=100 \mathrm{mHz}$.

Compact binaries containing stellar or intermediate mass black holes, white dwarfs or neutron stars could emit monochromatic GWs in the early inspiral phase which are detectable by space-based detectors and the corresponding frequency evolution is negligible during the mission of the detector. In the lowest order quadrupole approximation, the two polarizations of monochromatic GWs with the frequency $f_{0}$ are

$$
\begin{aligned}
& h_{+}=\mathcal{A}\left[1+(\vec{L} \cdot \hat{\omega})^{2}\right] \exp \left(2 \pi i f_{0} t+i \phi_{0}\right), \\
& h_{\times}=2 i \mathcal{A} \vec{L} \cdot \hat{\omega} \exp \left(2 \pi i f_{0} t+i \phi_{0}\right),
\end{aligned}
$$

where $\mathcal{A}=2 M_{1} M_{2} /\left(r d_{L}\right)$ is the amplitude, $M_{1}$ and $M_{2}$ are masses of the binary components, $r$ is the distance between them, $d_{L}$ is the luminosity distance between the source and the observer, $\vec{L}$ is the unit vector for the binary's orbital angular momentum, and $\phi_{0}$ is the initial phase. We focus mainly on the following seven parameters of the monochromatic GW signal included in Eq. (8)

$$
\boldsymbol{\theta}=\left\{\theta_{s}, \phi_{s}, \theta_{L}, \phi_{L}, \mathcal{A}, \phi_{0}, f_{0}\right\}
$$

These parameters describe a monochromatic source by the source direction $\left(\theta_{s}, \phi_{s}\right)$, the direction of the binary's orbital angular momentum $\left(\theta_{L}, \phi_{L}\right)$, the amplitude $\mathcal{A}$, the initial phase $\phi_{0}$ and frequency $f_{0}$. For monochromatic sources there is almost no frequency evolution, by using the Parseval's theorem [31, 46], the FIM becomes

$$
\begin{aligned}
\Gamma_{i j} & =\sum_{\alpha}\left(\frac{\partial H_{\alpha}}{\partial \theta_{i}} \mid \frac{\partial H_{\alpha}}{\partial \theta_{j}}\right) \\
& =\sum_{\alpha}\left[\frac{4}{S_{n, \alpha}\left(f_{0}\right)}\left(\int_{0}^{\infty} \partial_{i} H_{\alpha}(f) \partial_{j} H_{\alpha}^{*}(f) d f\right)\right] \\
& =\sum_{\alpha}\left[\frac{2}{S_{n, \alpha}\left(f_{0}\right)}\left(\int_{-\infty}^{\infty} \partial_{i} H_{\alpha}(t) \partial_{j} H_{\alpha}^{*}(t) d t\right)\right],
\end{aligned}
$$

where $\theta_{i}$ is the $i$ th parameter and $\partial_{i} H_{\alpha}=\partial H_{\alpha} / \partial \theta_{i}$. The covariance matrix of the parameters is

$$
\sigma_{i j}=\left\langle\Delta \theta^{i} \Delta \theta^{j}\right\rangle \approx\left(\Gamma^{-1}\right)_{i j} .
$$

For a detected source with a significant SNR (a threshold of $\rho \geq 7$ ), the angular uncertainty of the sky localization is evaluated as

$$
\Delta \Omega_{s} \equiv 2 \pi\left|\cos \theta_{s}\right| \sqrt{\sigma_{\theta_{s} \theta_{s}} \sigma_{\phi_{s} \phi_{s}}-\sigma_{\theta_{s} \phi_{s}}^{2}} .
$$

The signal in the detector coordinate system is $H(t)=$ $F^{+} h_{+}(t)+F^{\times} h_{\times}(t)+\hat{n}_{\alpha}(t)$. In practice, for space-based detectors, we often work in the heliocentric coordinate system. The translational motion of the center of the detector around the Sun leads to an extra phase modulation factor

$$
e^{i \phi_{D}(t)}=e^{2 \pi i f_{0} R \cos \theta_{s} \cos \left(2 \pi t / T-\phi_{s}-\phi_{\alpha}\right) / c},
$$

where $\phi_{\alpha}$ is the ecliptic longitude of the detector $\alpha$ at $t=0$, the period $T$ of the rotation is 1 year and the radius $\mathrm{R}$ of the orbit is $1 \mathrm{AU}$. Therefore, the signal in the heliocentric coordinate system is

$$
H(t)=\left[F^{+}(t) h_{+}(t)+F^{\times}(t) h_{\times}(t)\right] e^{i \phi_{D}(t)} .
$$

Since the phase modulation is very large in most cases and contains the information about the source location, this effect is very important for source localization and it is the same for LISA, Taiji and TianQin. From Eq. (13), we get

$$
\begin{aligned}
\delta f_{0} & =\left|\frac{1}{2 \pi} \partial_{t} \phi_{D}(t)\right| \\
& =f_{0} \cos \theta_{s}\left|\sin \left(2 \pi t / T-\phi_{s}-\phi_{\alpha}\right)\right| \frac{2 \pi R}{c T} \\
& \sim f_{0} \frac{v}{c},
\end{aligned}
$$

so the periodic phase modulation spreads the measured power of the monochromatic signal of frequency $f_{0}$ over a range $f_{0}(1 \pm v / c)$ and the spread depends on the direction of the source.

In addition to the Doppler modulation imposed by the rotation of the center of the detector around the Sun, the motion of the spacecrafts with respect to the detector center, the orientation change of the detector plane, the arm length and the noises of the detector also affect the accuracy of sky localization. In the next section, we analyze how much influences these effects have on the angular resolution of the detector in detail. 


\section{THE EFFECT OF DIFFERENT CONSTELLATION ON SKY LOCALIZATION}

LISA mission is proposed as an equilateral triangle constellation with sides of $2.5 \times 10^{6} \mathrm{~km}$ to detect low frequency GWs. The constellation has an inclination angle of $60^{\circ}$ with respect to the ecliptic plane and trails the Earth by about $20^{\circ}$. The inclination angle ensures the spacecrafts to keep the geometry of an equilateral triangle throughout the mission. As shown in Fig. 2, the normal vector of the detector plane rotates around the normal vector of the ecliptic plane and forms a cone with $60^{\circ}$ half opening angle in one year. Taiji has similar configuration to LISA except that its arm length is $3 \times 10^{6}$ $\mathrm{km}$. TianQin is an equilateral triangle constellation with sides of $1.73 \times 10^{5} \mathrm{~km}$ designed to orbit the Earth with the period of 3.65 days. The normal vector of the detector plane points to the direction of RX J0806.3+1527 with the latitude $\theta_{t q}=-4.7^{\circ}$ and the longitude $\phi_{t q}=120.5^{\circ}$ as shown in Fig. 2. The centers of LISA, Taiji, and TianQin all follow the Earth-like orbit. Three spacecrafts of the detectors are connected with each other by lasers in a form of equilateral triangle as shown in Fig. 3, we label three spacecrafts as $\mathrm{SC} 1 / 2 / 3$, three arms as $1 / 2 / 3$ and their lengths as $L_{1} / L_{2} / L_{3}$. We refer to the Michelson interferometer formed by the arms 1 and 2 as detector I and the Michelson interferometer formed by the arms 2 and 3 as detector II. We use both detectors I and II to determine the sky localization, so $\alpha=\mathrm{I}$ and II in Eq. (10).

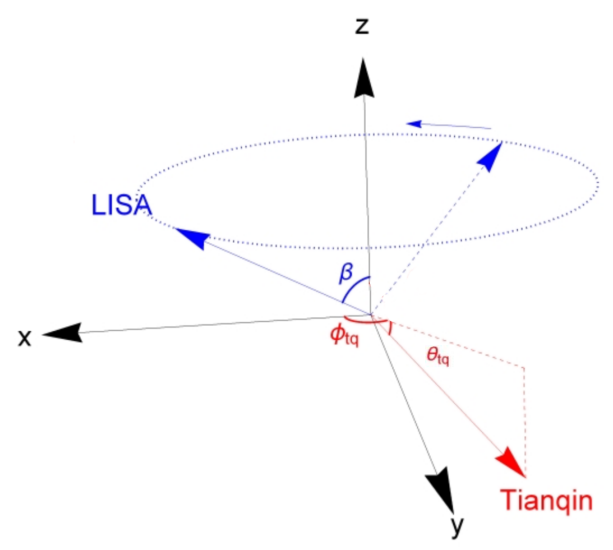

FIG. 2. The normal vectors of the detector planes for TianQin and LISA. Illustration from [47].

As analyzed above, there are four different factors which influence the detectors' localization precision. To disclose the effect of each factor on the source localization and compare its role in different detectors, we assume the other three factors the same in different detectors. Because we consider monochromatic sources, the noise power at the source frequency $f_{0}$ appears linearly in the Fisher matrix (10), so $S_{n}\left(f_{0}\right)$ can be eliminated by normalizing the result to a fixed SNR [31]. In other

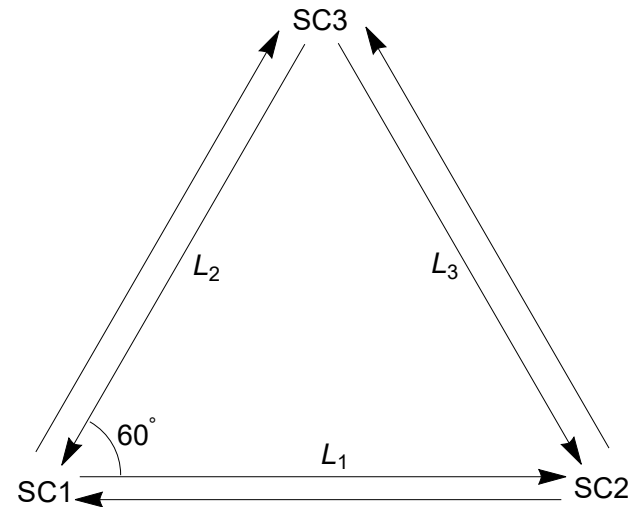

FIG. 3. The orientation of three arms in the detector plane.

words, we assume that $S_{n}\left(f_{0}\right)=1$ for all fiducial detectors so that the effect of each factor of the constellation on the source localization can be easily understood. Then we just multiply the ratio of $S_{n}\left(f_{0}\right)$ to the result when we compare the sky localization of LISA and TianQin. Before we start to compute the localization accuracy of detectors, we must give the parameters of sources and ensure that they could be detected. Thus we fix the SNR of all sources for the detector $\mathrm{R}$ (will be defined below) to be 7 , and then we derive the amplitudes $\mathcal{A}$ of sources from this SNR. This means that the amplitudes of sources being considered are different for different detectors. For a complimentary comparison, we present the results on the effect of different constellation by taking the same source (i.e., the sources have the same distance and masses) for different constellation in Appendix B. For this setting, perhaps some sources can not be detected by other detectors due to small SNR, we can increase the amplitudes of all sources by the same factor so that all sources can be detected by all detectors. Note that the relative angular resolution between detectors remains unchanged although the values of angular resolutions become smaller for each detector. The other parameters are fixed as $\theta_{L}=1.0$ and $\phi_{L}=\phi_{0}=0$. The mission time or the observation time $T_{o}$ is set to be one year. We simulate 3600 sources uniformly distributed in the sky with $-\pi / 2<\theta_{s}<\pi / 2$ and $-\pi<\phi_{s}<\pi$. We analyze the source localization accuracy at three frequencies $10^{-3} \mathrm{~Hz}, 10^{-2} \mathrm{~Hz}$ and $10^{-1} \mathrm{~Hz}$. The three frequencies stand for low, medium and high frequencies relative to the transfer frequency $f_{s}^{*}=c /\left(2 \pi L_{s}\right)=0.02 \mathrm{~Hz}$ in Eq. (5). 


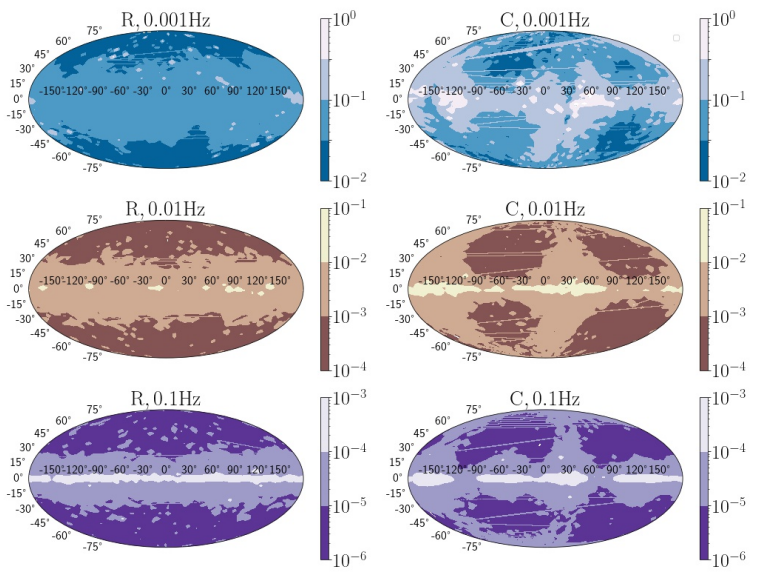

FIG. 4. The sky map of angular resolutions $\Delta \Omega_{S}$ of sources from different directions in the unit of steradian (1 steradian is 3000 square degrees) for the fiducial detectors $\mathrm{R}$ and $\mathrm{C}$. The horizontal axis represents the longitude $\phi_{s}$ and the vertical axis represents the latitude $\theta_{s}$. The left panel is for the detector $\mathrm{R}$ and the right panel is for the detector $\mathrm{C}$. From top to bottom, the frequencies of monochromatic sources are $10^{-3} \mathrm{~Hz}, 10^{-2} \mathrm{~Hz}$ and $10^{-1} \mathrm{~Hz}$.

\section{A. The rotation effect}

One major difference between TianQin and LISA/Taiji is the orientation of the detector plane. Naively we expect that the modulation of the signal caused by the rotation of the detector plane increases the accuracy of source localization for LISA and Taiji, and the precision of sky localization for TianQin becomes less without this modulation effect. To evaluate the effect of this time-changing orientation of the detector plane on sources' angular resolutions, we construct two fiducial GW detectors with the same arm length, noise curve and rotation period of the spacecrafts. The first detector is like LISA except that its arm length is $3.7 \times 10^{9} \mathrm{~m}$, and we call it detector $\mathrm{R}$. The second detector is like TianQin except that its arm length is $3.7 \times 10^{9} \mathrm{~m}$ and its rotation period around the Earth is one year, and we call it detector C. The detailed orbit equations for the detectors $\mathrm{R}$ and $\mathrm{C}$ are presented in Appendix A. For the arm length $L=3.7 \times 10^{9} \mathrm{~m}$, the transfer frequency is $f^{*}=c /(2 \pi L)=0.013 \mathrm{~Hz}$.

The results of angular resolutions at the frequencies $f_{0}=10^{-1} \mathrm{~Hz}, 10^{-2} \mathrm{~Hz}$ and $10^{-3} \mathrm{~Hz}$ for the detectors $\mathrm{R}$ and $\mathrm{C}$ are shown in Figs. 4, 5 and 6. We show the sky map of the angular resolution for the detectors $\mathrm{R}$ and $\mathrm{C}$ in Fig. 4, the cumulative histograms of the angular resolution in Fig. 5 and the ratios of the mean and median angular resolutions between the detectors $\mathrm{C}$ and $\mathrm{R}$ in Fig. 6. The mean and median values of angular resolutions are summarized in Table I. From Figs. 5 and 6, we see that the effect of the detector's time-varying orientation decreases as the frequency of GWs increases, the improvement is only a factor of 2.4 for the mean angular resolution and a factor of 1.5 for the median angular res-
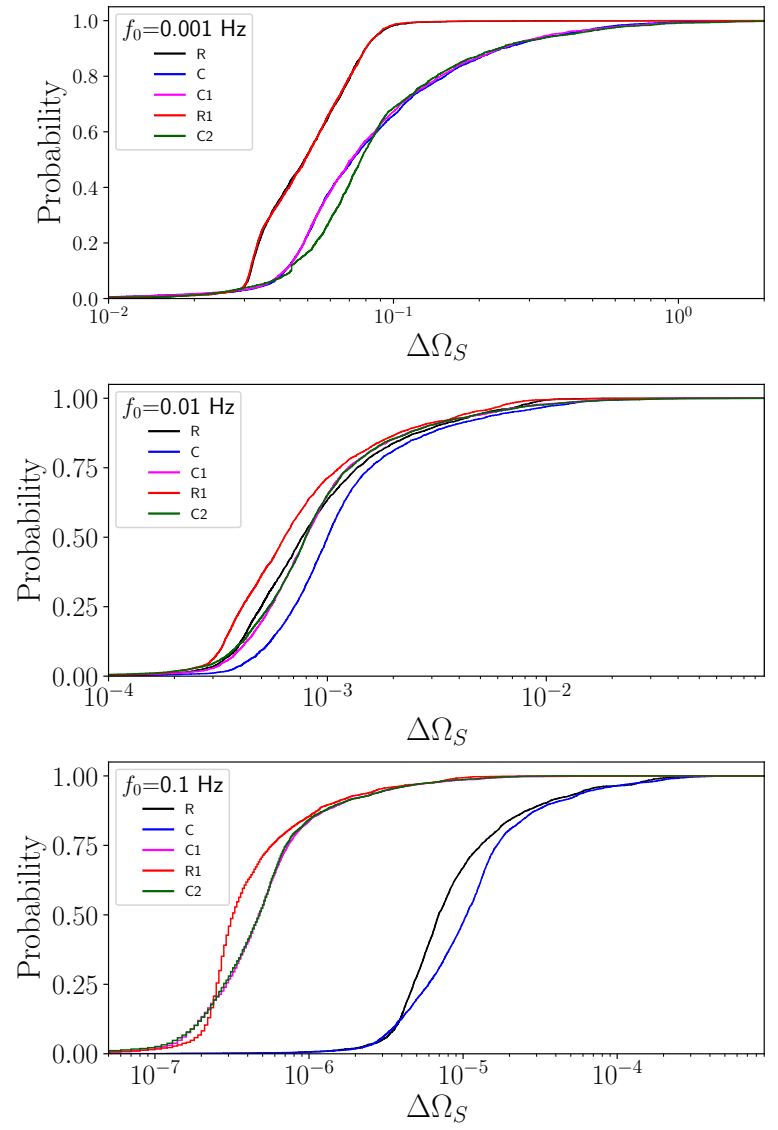

FIG. 5. Cumulative histograms of sky localization estimations $\Delta \Omega_{S}$ for different detectors.

\begin{tabular}{|c|c|c|c|c|c|}
\hline$f_{0}(\mathrm{~Hz})$ & $\mathrm{R}$ & $\mathrm{C}$ & $\mathrm{C} 1$ & $\mathrm{C} 2$ & $\mathrm{R} 1$ \\
\hline & \multicolumn{5}{|c|}{ Mean Value } \\
\hline $10^{-1}$ & $2.4 \times 10^{-5}$ & $2.7 \times 10^{-5}$ & $1.5 \times 10^{-6}$ & $1.2 \times 10^{-6}$ & $1.1 \times 10^{-6}$ \\
\hline $10^{-2}$ & $1.8 \times 10^{-3}$ & $2.3 \times 10^{-3}$ & $2.0 \times 10^{-3}$ & $2.1 \times 10^{-3}$ & $1.6 \times 10^{-3}$ \\
\hline $10^{-3}$ & $5.9 \times 10^{-2}$ & $1.4 \times 10^{-1}$ & $1.5 \times 10^{-1}$ & $1.7 \times 10^{-1}$ & $5.9 \times 10^{-2}$ \\
\hline \multicolumn{5}{|c|}{ Median Value } \\
\hline $10^{-1}$ & $7.0 \times 10^{-6}$ & $1.0 \times 10^{-5}$ & $4.7 \times 10^{-7}$ & $4.8 \times 10^{-7}$ & $4.2 \times 10^{-7}$ \\
\hline $10^{-2}$ & $7.6 \times 10^{-4}$ & $1.0 \times 10^{-3}$ & $7.9 \times 10^{-4}$ & $7.9 \times 10^{-4}$ & $5.0 \times 10^{-4}$ \\
\hline $10^{-3}$ & $4.8 \times 10^{-2}$ & $7.1 \times 10^{-2}$ & $7.1 \times 10^{-2}$ & $7.6 \times 10^{-2}$ & $4.8 \times 10^{-2}$ \\
\hline
\end{tabular}

TABLE I. The mean and median values of angular resolutions $\Delta \Omega_{S}$ for different detectors.

olution at the frequency $f_{0}=1 \mathrm{mHz}$ with the amplitude modulation caused by the detector's time-varying orientation. However, the median and mean values of angular resolutions of the detector as usually presented in the literature are incomplete because we don't know where the poorly localized regions are. Thus we also present the sky map of angular resolutions in Fig. 4.

From Fig. 4, we see that there are some regions in which the uncertainties of the angular resolutions are large, but the locations of these regions cannot be seen in the histogram. As the frequencies of the monochro- 
matic GW sources increase, the uncertainties of the angular resolutions become smaller. For the detector R, at the low frequency $f_{0}=10^{-3} \mathrm{~Hz}$, sky localization uncertainties $\Delta \Omega_{S}$ are around 0.01 to 0.1 steradians across the sky. Comparing with sources from other directions, $\Delta \Omega_{S}$ are more than two orders of magnitude larger for sources along the equatorial plane with the latitude $\theta_{s}=0$ in the heliocentric coordinate system at the medium and high frequencies. For the detector $\mathrm{C}$, the angular resolutions for sources along the detector plane $\left(\phi_{s}\right.$ is around $-150^{\circ}$ and $30^{\circ}$ ) and the equatorial plane in the heliocentric coordinate system are much worse.

In Fourier space, the Doppler effect spreads the measured power of the monochromatic signal of frequency $f_{0}$ over a range $f_{0}(1 \pm v / c)$, where the speed of the motion of the detector's center around the Sun $v \sim 2 \pi R / T=$ $3 \times 10^{4} \mathrm{~m} / \mathrm{s}$, so the sky localization is better when $f_{0}$ increases and the frequency spread caused by the Doppler effect at $f_{0}=10^{-3} \mathrm{~Hz}$ is $\delta f=f_{0} v / c \sim 10^{-7}$. The Doppler effect on the angular resolution for monochromatic sources is [32]

$$
\begin{aligned}
\delta \Omega & =\frac{1}{f^{2} \pi R_{s}^{2} \rho^{2} \sqrt{1-6 x^{2}}}\left\{\frac{6 R_{s}^{2}}{T_{o}^{2}} \csc ^{2}\left(\theta_{s}\right) \cos \left(\theta_{s}\right)^{2}\right. \\
& \left.-12 \frac{R_{s}}{T_{0}} x \cot \left(\theta_{s}\right) \sin \left(\phi_{s}\right) \csc \left(\theta_{s}\right)+\csc ^{2}\left(\theta_{s}\right)\right\}^{1 / 2} \\
& \approx 0.02\left(1 \mathrm{mHz} / f_{0}\right)^{2}(10 / \rho)^{2}\left|\sin \left(\theta_{s}\right)\right|^{-1},
\end{aligned}
$$

where the distance between the Earth and the Sun $R_{s}=$ $1 \mathrm{AU}$, the observational time $T_{o}=1$ year and $x=1 / \pi$. It is easy to see that the sky localization becomes better as frequency increases and it depends on the direction of the source. For sources along the equatorial plane, the latitude $\theta_{s}=0$, the sky localization becomes worse, so the Doppler modulation has the equatorial pattern [32]. On the other hand, the time-varying orientation of the detector's plane spreads the power over a range $f_{0} \pm 2 / T=f_{0} \pm 6.3 \times 10^{-8}$ with $T=1$ year (i.e., the frequency spread $\left.\delta f=2 / T=6.3 \times 10^{-8}\right)$, and it is independent of the source location. At $f_{0}=10^{-3} \mathrm{~Hz}$, the frequency spread caused by the Doppler modulation is $\delta f \sim 10^{-7}$, so the frequency spread caused by the amplitude modulation is the same order as that caused by the Doppler modulation, this explains why the angular resolution for the detector $\mathrm{R}$ is almost the same for sources from any direction, especially good angular resolutions along the equatorial plane. As the frequency of GWs increases, the Doppler effect dominates. Therefore the angular resolutions for the detector $\mathrm{R}$ and $\mathrm{C}$ are almost the same at medium and high frequencies, and the sky localization becomes worse for sources along the equatorial plane. For the detector $\mathrm{C}$, if sources are located along the detector's plane, then the SNR is smaller and the sky localization becomes worse.

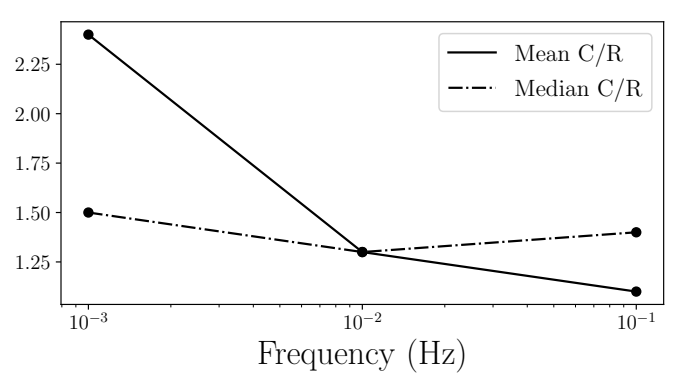

FIG. 6. The ratios of the mean and median values of the angular resolutions between the detectors $\mathrm{R}$ and $\mathrm{C}$.

\section{B. The effect of the arm length}

Another difference between TianQin and LISA/Taiji is the distance between spacecrafts. When the wavelength of GWs is larger than the arm length, i.e., $f_{0}<f_{*}$, the transfer function (5) is almost equal to 1 , so the transfer function is independent of GWs' frequency and the effect of the arm length on the transfer function is negligible. If $f_{0}>f_{*}$, then the frequency dependence of the transfer function deteriorates the response of the detector. To evaluate the influence of the detector's arm length on angular resolutions, we devise two more fiducial GW detectors with the same noise curve, rotation period of the spacecrafts and orientation of the detector plane but with different arm lengths. The third detector is like the detector $\mathrm{C}$ except that its arm length is $1.7 \times 10^{8} \mathrm{~m}$, and we call it detector $\mathrm{C} 1$. For the detector $\mathrm{C} 1$, we assume a fiducial Earth with the right mass to provide the orbit. Since the mass of the Earth does not affect the performance of the detector, so there is no problem with this assumption for the purpose of the discussion on the effect of the arm length. The fourth detector is like the detector $\mathrm{R}$ except that its arm length is $1.7 \times 10^{8} \mathrm{~m}$, and we call it detector R1. For the arm length $L_{t}=1.7 \times 10^{8}$ $\mathrm{m}$, the transfer frequency is $f_{t}^{*}=c / 2 \pi L_{t}=0.28 \mathrm{~Hz}$. The detailed orbit equations for the detectors $\mathrm{C} 1$ and $\mathrm{R} 1$ are described in Appendix A. The effect of the arm length can be analyzed by comparing the angular resolutions for either the detectors $\mathrm{R}$ and $\mathrm{R} 1$ or $\mathrm{C}$ and $\mathrm{C} 1$.

Figure 7 shows the sky map of angular resolutions for the detectors R1 and C1. Because of the Doppler effect, the angular resolution is better at higher frequency. Since the frequencies $f_{0}=1 \mathrm{mHz}$ and $f_{0}=10 \mathrm{mHz}$ are smaller than the transfer frequencies $f^{*}=13 \mathrm{mHz}$ (for the arm length $L=3.7 \times 10^{9} \mathrm{~m}$ ) and $f_{t}^{*}=0.28 \mathrm{~Hz}$ (for the arm length $L_{t}=1.7 \times 10^{8} \mathrm{~m}$ ), so the angular resolutions are similar for either the detectors $\mathrm{R}$ and $\mathrm{R} 1$ or the detectors $\mathrm{C}$ and $\mathrm{C} 1$ at $f_{0}=1 \mathrm{mHz}$ and $f_{0}=10 \mathrm{mHz}$ as shown in Figs. 5 and 8. At the frequency $f_{0}=0.1 \mathrm{~Hz}$, the wavelength of GWs is comparable with the arm length $L_{t}=1.7 \times 10^{8} \mathrm{~m}$ and less than the arm length $L=$ $3.7 \times 10^{9} \mathrm{~m}$, so angular resolutions of the detectors R1 and $\mathrm{C} 1$ are better than those of the detectors $\mathrm{R}$ and $\mathrm{C}$, as 

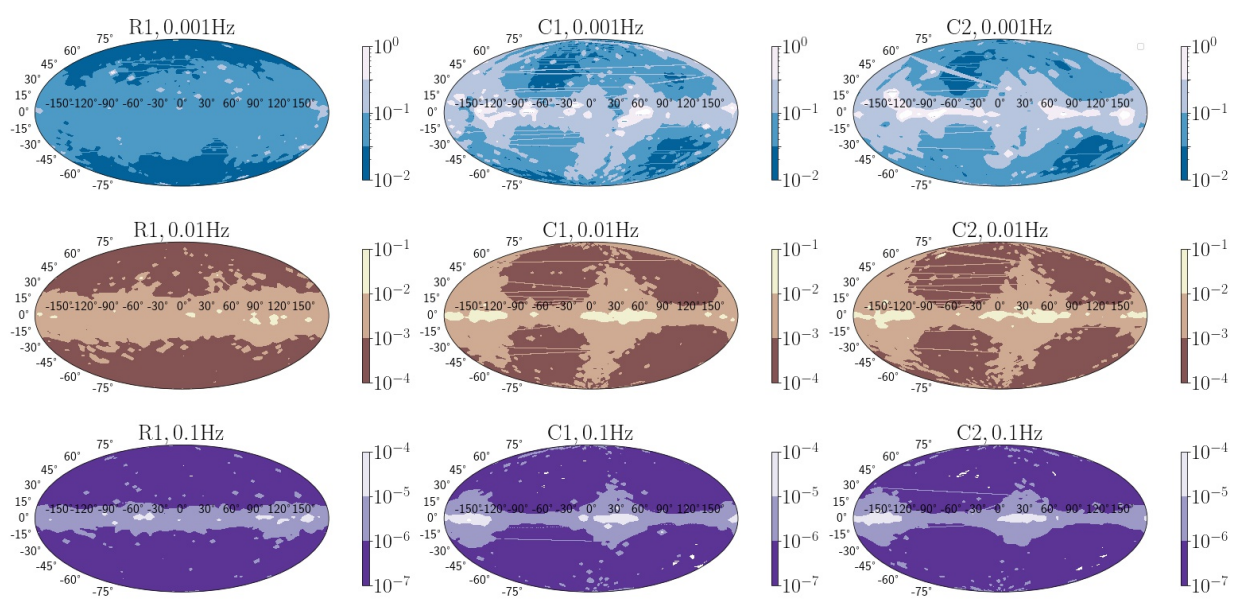

FIG. 7. The sky map of angular resolutions $\Delta \Omega_{S}$ for the detectors R1, C1 and C2. The left panel is for the detector R1, the middle panel is for the detector $\mathrm{C} 1$ and the right panel is for the detector $\mathrm{C} 2$. From top to bottom, the frequencies are $10^{-3}$ $\mathrm{Hz}, 10^{-2} \mathrm{~Hz}$ and $10^{-1} \mathrm{~Hz}$.

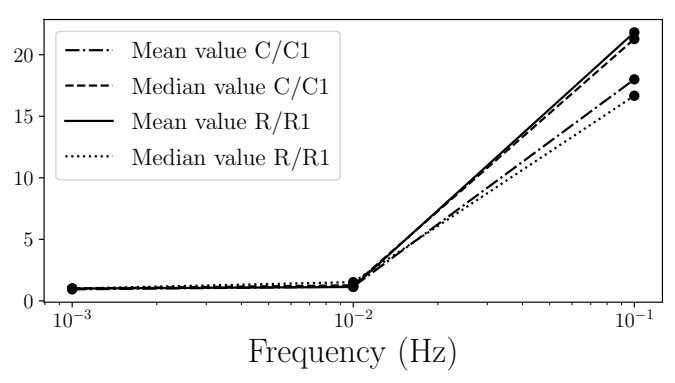

FIG. 8. The ratios of the mean and median values of angular resolutions between the detectors $\mathrm{R}$ and $\mathrm{R} 1$, and the detectors $\mathrm{C}$ and $\mathrm{C} 1$.

shown in Figs. 5 and 8. We also summarize the mean and median values of angular resolutions for these detectors in Table I. These results tell us that the effect of the arm length is almost the same for the heliocentric and geocentric constellations and the factor of improvement on the angular resolution is about $L / L_{t}=22$ at $f_{0}=0.1$ Hz. As expected, TianQin is more sensitive than LISA at frequencies $f_{0} \gtrsim 10 \mathrm{mHz}$.

\section{Rotation period of the spacecrafts}

The rotation period of the spacecrafts for TianQin is 3.65 days and it is 1 year for LISA/Taiji. To evaluate the effect of the rotation period of the spacecrafts on sources' angular resolutions, we devise another fiducial GW detector which is like the detector $\mathrm{C} 1$ except that its rotation period is 3.65 days, and we call it detector $\mathrm{C} 2$. The detailed orbit equations for the detector $\mathrm{C} 2$ are given in Appendix A. Without specifying the noises, the detector $\mathrm{C} 2$ is the same as TianQin.

Figures 5 and 7 show the histograms and the sky map of angular resolutions for the detector $\mathrm{C} 2$, respectively. The mean and median values of angular resolutions for the detector $\mathrm{C} 2$ are given in Table. I. These results show that the influence of the rotation period of the spacecrafts is almost negligible. Because of the cylindrical symmetry of the rotation, the rotations of the spacecrafts around the fixed axis have no effect on angular resolutions in one period.

In the real geocentric orbit, the rotation period depends on the arm length. If we increase the arm length, the detector's sensitivity increases and the detector is more sensitive at lower frequency.

\section{LISA-TIANQIN NETWORK}

At the frequency $f_{0}=1 \mathrm{mHz}$, the contribution of the amplitude modulation due to the time changing orientation of the detector plane with a period of one year to the accuracy of the angular resolution is comparable to that of the Doppler modulation due to the motion of the center of the detector around the Sun, so the amplitude modulation helps LISA and Taiji not only get better angular resolution, but also enlarge the sky coverage because the amplitude modulation is independent of the sources' directions. At higher frequencies when the wavelength of GWs is larger than the detector's arm length, the frequency dependent transfer function deteriorates the SNR registered in the detector, so we expect that the accuracy of the sky localization for TianQin is better because of its shorter arm length. Now we discuss the sky localization estimations of LISA, TianQin and the LISA-TianQin network. Since Taiji and LISA have similar constellation, so we discuss LISA only. We fix the SNR of all sources for LISA to be 7, then we derive the amplitudes $\mathcal{A}$ of sources from this fixed SNR, and calculate the angular resolutions of LISA, TianQin and the 
LISA-TianQin network.

We show the histograms of angular resolutions in Fig. 9 and summarize the mean and median values of angular resolutions in Table II. At the frequency $f_{0}=10^{-3}$ $\mathrm{Hz}$, LISA's angular resolution is roughly 50 times better than TianQin because its noise $S_{n}\left(f_{0}\right)$ is about 23 times smaller and the amplitude modulation contributes another factor of 2.4, the angular resolution of the combined network is almost the same as LISA. At the frequency $f_{0}=10^{-2} \mathrm{~Hz}$, LISA's angular resolution is about 1.4 times better than TianQin because TianQin's noise $S_{n}\left(f_{0}\right)$ is about 1.2 times smaller and its rotation effect is about 1.2 times smaller, and the network's angular resolution is a little better than LISA. At the frequency $f_{0}=0.1 \mathrm{~Hz}$, the frequency dependent transfer function reduces LISA's sensitivity by a factor about 10 , so TianQin's angular resolution becomes about 10 times better than LISA. Therefore, the angular resolution of the combined LISA-TianQin network spans over the frequency ranges $1-100 \mathrm{mHz}$ and it reaches $10^{-6}$ steradians at $f_{0}=0.1 \mathrm{~Hz}$.

In Fig. 10, we plot the sky map of angular resolutions for LISA, TianQin and their combined network. At $f_{0}=1 \mathrm{mHz}$, the amplitude and Doppler modulations contribute to LISA's angular resolutions, so LISA's angular resolutions are almost the same across the sky. At higher frequencies, the contribution of the amplitude modulation is negligible and only the Doppler modulation matters. For sources along the equatorial plane, the angular resolution is the worst for both LISA and TianQin at the frequencies $f_{0}=10 \mathrm{mHz}$ and $f_{0}=100$ $\mathrm{mHz}$. For TianQin, the worst angular resolution also occurs for sources from the directions with $\phi_{s}=30^{\circ}$ and $\phi_{s}=-150^{\circ}$. Fig. 10 shows that the combined network enlarges the sky coverage in addition to the slight improvement on angular resolutions at $f_{0}=10 \mathrm{mHz}$ and $f_{0}=100 \mathrm{mHz}$.

\section{CONCLUSION}

The detector's noises, arm length, time-changing orientation, motion around the Sun are four main factors that influence the accuracy of source localization for spacedbased GW observatories. The detector's noises and arm length affect the angular resolution through SNR. The Doppler modulation on the amplitude and phase of the GW signal imposed by the translational motion of the detector's center around the Sun carries the position information of the source. For LISA and Taiji, the amplitude of GW signal is further modulated by the annual rotation of the detector's orientation. Both the Doppler and amplitude modulations spread the power of the monochromatic GWs over a frequency range in Fourier space. The amplitude modulation improves the accuracy of the sky localization below several $\mathrm{mHz}$ and the improvement is the same for sources from different directions. The effect of the Doppler modulation on the angular resolution be-
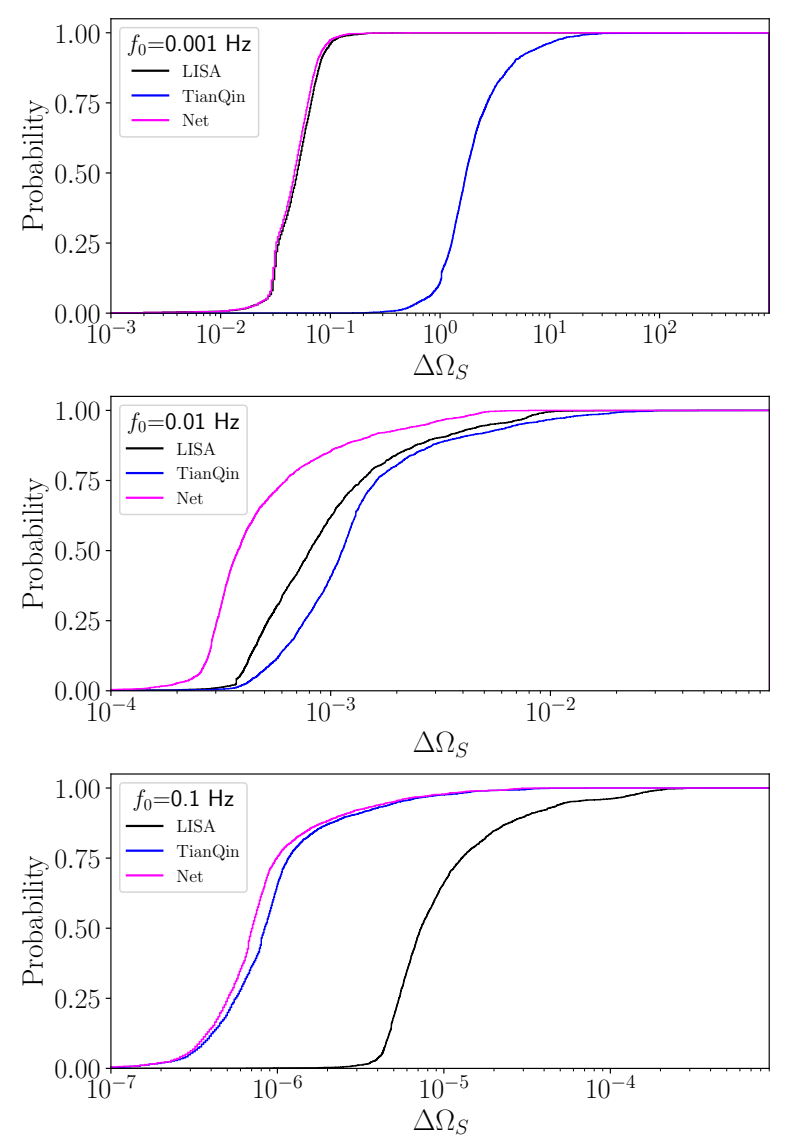

FIG. 9. Cumulative histograms of sky localization uncertainties $\Delta \Omega_{S}$ for LISA, TianQin and their combined network.

comes better as the frequency of the monochromatic GW source increases, and this effect depends on the direction of the source. For sources along the plane with $\theta_{s}=\pi / 2$ in the heliocentric coordinate system, the improvement by the Doppler modulation on the angular resolution is the largest. For frequencies above approximately $5 \mathrm{mHz}$, the contribution of the amplitude modulation is negligible and only the Doppler modulation matters. Therefore, TianQin's angular resolution is not affected much due to the lack of amplitude modulation. By pointing to the specific source, TianQin has some blind spots, its angular resolution is not good for sources from the directions with $\phi_{s}$ around $30^{\circ}$ or $-150^{\circ}$.

At the frequency $f_{0}=1 \mathrm{mHz}$, the contribution of the amplitude modulation is comparable to that of the Doppler modulation, so the amplitude modulation helps LISA and Taiji not only get better angular resolution, but also enlarge the sky coverage. At higher frequencies when the wavelength of GWs is larger than the detector's arm length, the frequency dependent transfer function deteriorates the SNR registered in the detector. For the monochromatic sources with the frequency $f_{s}^{*}=c /\left(2 \pi L_{s}\right)<f_{0}<f_{t}^{*}=c /\left(2 \pi L_{t}\right)$, TianQin's angular resolution is better than LISA's by a factor of 


\begin{tabular}{|c|c|c||c|c||c|c|}
\hline & \multicolumn{2}{|c||}{ LISA } & \multicolumn{2}{c||}{ TianQin } & \multicolumn{2}{c|}{ Network } \\
\hline$f_{0}(\mathrm{~Hz})$ & Mean & Median & Mean & Median & Mean & Median \\
\hline $10^{-1}$ & $2.3 \times 10^{-5}$ & $7.4 \times 10^{-6}$ & $2.1 \times 10^{-6}$ & $8.3 \times 10^{-7}$ & $1.8 \times 10^{-6}$ & $7.1 \times 10^{-7}$ \\
\hline $10^{-2}$ & $2.0 \times 10^{-3}$ & $8.2 \times 10^{-4}$ & $2.5 \times 10^{-3}$ & $1.1 \times 10^{-3}$ & $8.9 \times 10^{-4}$ & $3.8 \times 10^{-4}$ \\
\hline $10^{-3}$ & $6.2 \times 10^{-2}$ & $5.03 \times 10^{-2}$ & 3.7 & 1.7 & $5.7 \times 10^{-2}$ & $4.9 \times 10^{-2}$ \\
\hline
\end{tabular}

TABLE II. The mean and median values of angular resolutions for monochromatic sources.
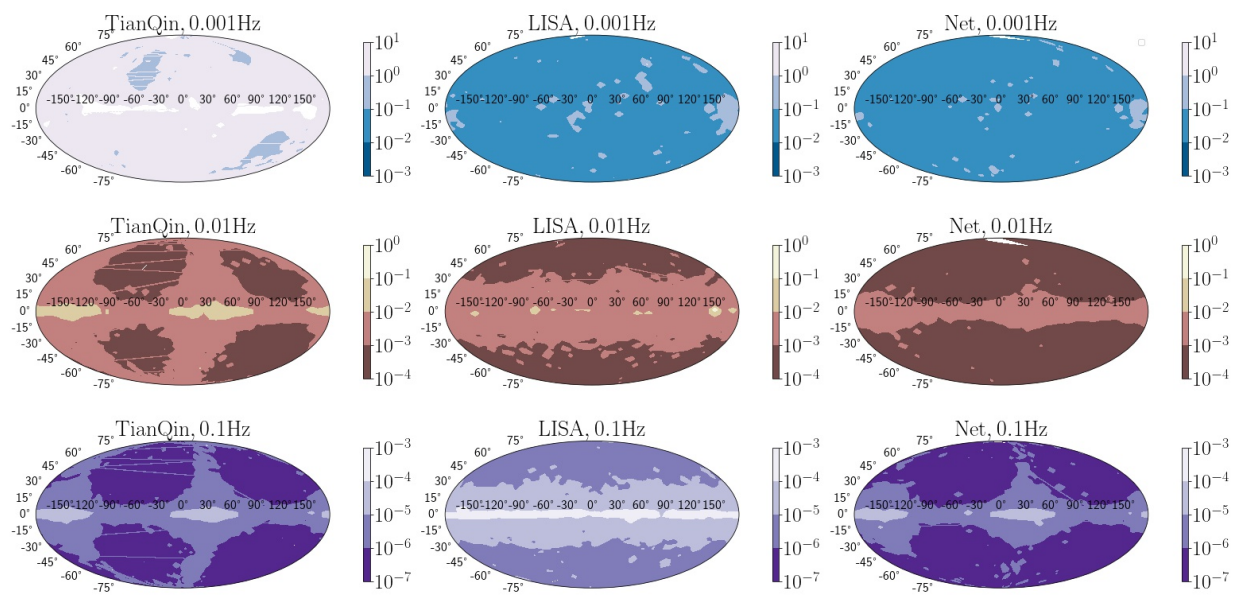

FIG. 10. The sky map of angular resolutions $\Delta \Omega_{S}$ for LISA, TianQin and their combined network at the frequencies $f_{0}=10^{-3}$ $\mathrm{Hz}, f_{0}=10^{-2} \mathrm{~Hz}$ and $f_{0}=10^{-1} \mathrm{~Hz}$.

roughly $S_{n, t} L_{s} /\left(S_{n, s} L_{t}\right)$, here $S_{n, s}$ and $L_{s}$ are LISA's noise curve and arm length, and $S_{n, t}$ and $L_{t}$ are TianQin's noise curve and arm length. Since LISA and Taiji have the best sensitivity at around $1 \mathrm{mHz}$, and TianQin is more sensitive at $10 \mathrm{mHz}$, the LISA-TianQin or TaiJiTianQin network have better ability of sky localization for sources in the frequency range $1-100 \mathrm{mHz}$ and the network has larger sky coverage for the angular resolution than the individual detector. By assuming $\mathrm{SNR}=7$ for LISA, the network's angular resolution is about 200 square degrees at $1 \mathrm{mHz}, 3$ square degrees at $10 \mathrm{mHz}$ and 0.005 square degrees at $100 \mathrm{mHz}$. The understanding of the effects of the amplitude and Doppler modulations on LISA/Taiji/TianQin can be used to optimize their constellations. In particular, the result may help TianQin to improve the design so that the equatorial pattern and

$$
\begin{aligned}
X_{n}^{T}(t)= & R_{T}\left[\cos \theta_{t q} \cos \phi_{t q} \sin \left(\alpha_{T n}-\alpha_{T 0}^{\prime}\right)+\sin \phi_{t q} \cos \left(\alpha_{T n}-\alpha_{T 0}^{\prime}\right)\right] \\
& +\frac{1}{2} e_{T} R_{T}\left[\sin \phi_{t q}\left(\cos 2\left(\alpha_{T n}-\alpha_{T 0}^{\prime}\right)-3\right)+\cos \theta_{t q} \cos \phi_{t q} \sin 2\left(\alpha_{T n}-\alpha_{T 0}^{\prime}\right)\right] \\
& +\frac{1}{4} e_{T}^{2} R_{T}\left[-6 \sin \phi_{t q} \cos \left(\alpha_{T n}-\alpha_{T 0}^{\prime}\right) \sin ^{2}\left(\alpha_{T n}-\alpha_{T 0}^{\prime}\right)+\cos \theta_{t q} \cos \phi_{t q}\left(3 \cos 2\left(\alpha_{T n}-\alpha_{T 0}^{\prime}\right)-1\right)\right] \\
& +R \cos \left(\alpha_{T}-\alpha_{T 0}\right)+\frac{1}{2} e R\left(\cos 2\left(\alpha_{T}-\alpha_{T 0}\right)-3\right)-\frac{3}{2} e^{2} R \cos \left(\alpha_{T}-\alpha_{T 0}\right) \sin ^{2}\left(\alpha_{T}-\alpha_{T 0}\right)+O\left(e^{3}, e_{1}^{3}\right),
\end{aligned}
$$




$$
\begin{aligned}
Y_{n}^{T}(t)= & R_{T}\left[\cos \theta_{t q} \sin \phi_{t q} \sin \left(\alpha_{T n}-\alpha_{T 0}^{\prime}\right)-\cos \phi_{t q} \cos \left(\alpha_{T n}-\alpha_{T 0}^{\prime}\right)\right] \\
& +\frac{1}{2} e_{T} R_{T}\left[-\cos \phi_{t q}\left(\cos 2\left(\alpha_{T n}-\alpha_{T 0}^{\prime}\right)-3\right)+\cos \theta_{t q} \sin \phi_{t q} \sin 2\left(\alpha_{T n}-\alpha_{T 0}^{\prime}\right)\right] \\
& +\frac{1}{4} e_{T}^{2} R_{T}\left[6 \cos \phi_{t q} \cos \left(\alpha_{T n}-\alpha_{T 0}^{\prime}\right) \sin ^{2}\left(\alpha_{T n}-\alpha_{T 0}^{\prime}\right)+\right. \\
& \left.\cos \theta_{t q} \sin \phi_{t q} \sin \left(\alpha_{T n}-\alpha_{T 0}^{\prime}\right)\left(3 \cos 2\left(\alpha_{T n}-\alpha_{T 0}^{\prime}\right)-1\right)\right] \\
& +R \sin \left(\alpha_{T}-\alpha_{T 0}\right)+\frac{1}{2} e R \sin 2\left(\alpha_{T}-\alpha_{T 0}\right)+\frac{1}{4} e^{2} R \sin \left(\alpha_{T}-\alpha_{T 0}\right)\left(e \cos 2\left(\alpha_{T}-\alpha_{T 0}\right)-1\right)+O\left(e^{3}, e_{1}^{3}\right) \\
& \quad Z_{n}^{T}(t)=-R_{T} \sin \theta_{t q} \sin \left(\alpha_{T n}-\alpha_{T 0}^{\prime}\right)-\frac{1}{2} e_{T} R_{T} \sin \theta_{t q} \sin 2\left(\alpha_{T n}-\alpha_{T 0}^{\prime}\right) \\
& -\frac{1}{4} e_{T}^{2} R_{T} \sin \theta_{t q} \sin \left(\alpha_{T n}-\alpha_{T 0}^{\prime}\right) \times\left[3 \cos 2\left(\alpha_{T n}-\alpha_{T 0}^{\prime}\right)-1\right]
\end{aligned}
$$

where $\left(\theta_{t q}=-4.7^{\circ}, \phi_{t q}=120.5^{\circ}\right)$ is the location of the source RX J0806.3+1527, $e_{T}$ is the eccentricity of the orbit which we set to be 0 in this paper, $e=0.0167$ is the eccentricity of the Earth's orbit, $R=1 \mathrm{AU}, \alpha_{T 0}$ and $\alpha_{T 0}^{\prime}$ are initial phases, $\alpha_{T}=2 \pi f_{m} t+\kappa_{0}, f_{m}=1 /(365$ days), $\alpha_{T n}=2 \pi f_{s c} t+2 \pi(n-1) / 3$. For the detector $\mathrm{C}$, the semimajor axis of the spacecraft's orbit $R_{T}=$ $3.7 \times 10^{6} \mathrm{~km}, f_{s c}=1 /(365$ days $)$. For the detector C1, $R_{T}=1.7 \times 10^{5} \mathrm{~km}, f_{s c}=1 /(365$ days $)$. For the detector $\mathrm{C} 2, R_{T}=1.7 \times 10^{5} \mathrm{~km}, f_{s c}=1 /(3.65$ days $)$.

\section{The orbits for the detectors $R$ and $R 1$}

The orbit $\vec{r}_{n}^{L}(t)=\left(X_{n}^{L}(t), Y_{n}^{L}(t), Z_{n}^{L}(t)\right)$ in the ecliptic coordinate system is

$$
\begin{gathered}
X_{n}^{L}(t)=R\left(\cos \alpha_{L n}+e_{L}\right) \cos \epsilon \cos \theta_{L n} \\
-R \sqrt{1-e_{L}^{2}} \sin \alpha_{L n} \sin \theta_{L n}, \\
Y_{n}^{L}(t)=R\left(\cos \alpha_{L n}+e_{L}\right) \cos \epsilon \sin \theta_{L n} \\
+R \sqrt{1-e_{L}^{2}} \sin \alpha_{L n} \cos \theta_{L n}, \\
Z_{n}^{L}(t)=R\left(\cos \alpha_{L n}+e_{L}\right) \sin \epsilon,
\end{gathered}
$$

where $e_{L}=\left(1+\frac{2}{\sqrt{3}} \sigma+\frac{4}{3} \sigma^{2}\right)^{\frac{1}{2}}-1, \epsilon=\arctan [\sigma /(1+$ $\sigma / \sqrt{3})], \sigma=L /(2 R), R \approx 1 \mathrm{AU}, \theta_{L n}=2 \pi(n-1) / 3$, $\alpha_{L n}+e_{L} \sin \alpha_{L n}=2 \pi f_{m} t-2 \pi(n-1) / 3-\alpha_{L 0}$ and $\alpha_{L 0}$ is the initial phase. The arm length $L=3.7 \times 10^{9} \mathrm{~m}$ for the detector $\mathrm{R}$ and $L=1.7 \times 10^{8} \mathrm{~m}$ for the detector $\mathrm{R} 1$.

\section{Appendix B: THE EFFECT DIFFERENT CONSTELLATION FOR SOURCES WITH FIXED AMPLITUDE}

In this section, instead of fixing the SNR, we fix the amplitude of the sources, i.e., we consider sources with the same distance and masses for different detectors. To consider monochromatic GWs with the frequency $\left(10^{-1}, 10^{-2}, 10^{-3}\right) \mathrm{Hz}$, we take the sources to be equal mass binary systems with the total mass $\left(6,3 \times 10^{2}, 10^{4}\right) M_{\odot}$ and the distance $\left(2.3,1.3 \times 10^{3}, 10^{4}\right)$ Mpc, respectively. The noise power $S_{n}\left(f_{0}\right)$ is chosen as $\left(3.6 \times 10^{-41}, 3.6 \times 10^{-41}, 4.1 \times 10^{-41}\right)$ at the frequency $\left(10^{-1}, 10^{-2}, 10^{-3}\right)$, respectively. The sky map of angular resolutions for the detectors $\mathrm{R}, \mathrm{C}, \mathrm{R} 1, \mathrm{C} 1$ and $\mathrm{C} 2$ are shown in Figs. 11 and 12 which are consistent with our conclusions $(\mathrm{SNR}=7)$.

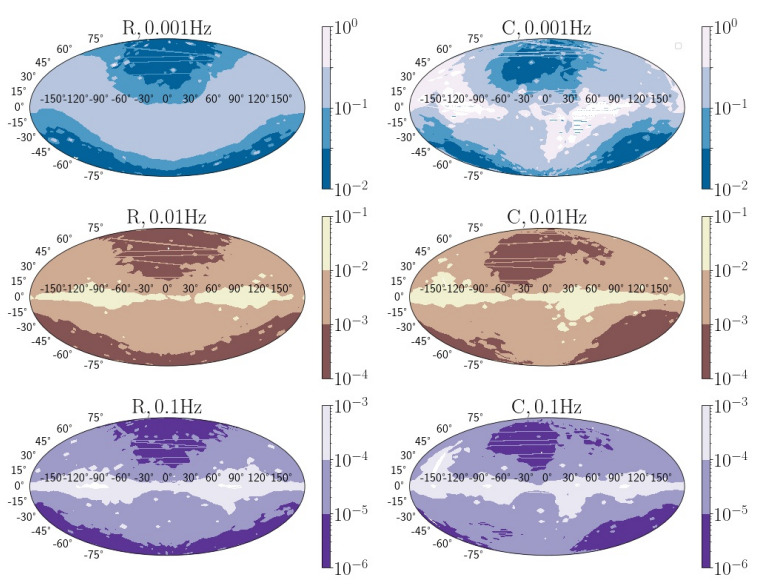

FIG. 11. The sky map of angular resolutions $\Delta \Omega_{S}$ for the fiducial detectors $\mathrm{R}$ and $\mathrm{C}$. For the same frequency, the sources have the same amplitude. The left panel is for the detector $\mathrm{R}$ and the right panel is for the detector C. From top to bottom, the frequencies of monochromatic sources are $10^{-3} \mathrm{~Hz}, 10^{-2}$ $\mathrm{Hz}$ and $10^{-1} \mathrm{~Hz}$.
[1] B. P. Abbott et al. (LIGO Scientific and Virgo Collaborations), Observation of Gravitational Waves from a Binary
Black Hole Merger, Phys. Rev. Lett. 116, 061102 (2016). 

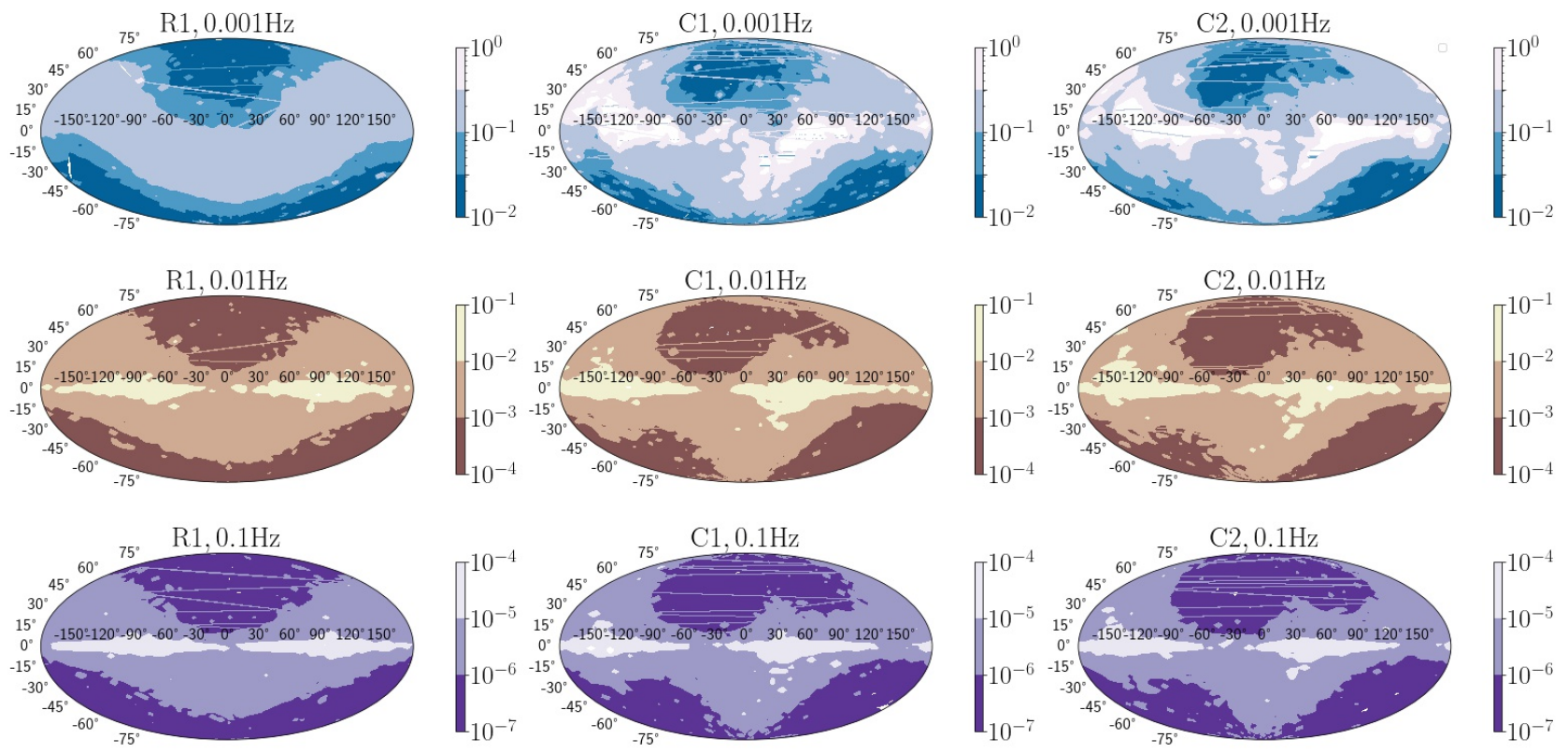

FIG. 12. The sky map of angular resolutions $\Delta \Omega_{S}$ for the detectors R1, C1 and C2. For the same frequency, the sources have the same amplitude The left panel is for the detector R1, the middle panel is for the detector $\mathrm{C} 1$ and the right panel is for the detector C2. From top to bottom, the frequencies are $10^{-3} \mathrm{~Hz}, 10^{-2} \mathrm{~Hz}$ and $10^{-1} \mathrm{~Hz}$.

[2] B. P. Abbott et al. (LIGO Scientific and Virgo Collaborations), GW150914: The Advanced LIGO Detectors in the Era of First Discoveries, Phys. Rev. Lett. 116, 131103 (2016).

[3] B. P. Abbott et al. (LIGO Scientific and Virgo Collaborations), GW151226: Observation of Gravitational Waves from a 22-Solar-Mass Binary Black Hole Coalescence, Phys. Rev. Lett. 116, 241103 (2016).

[4] B. P. Abbott et al. (LIGO Scientific and Virgo Collaborations), GW170104: Observation of a 50-Solar-Mass Binary Black Hole Coalescence at Redshift 0.2, Phys. Rev. Lett. 118, 221101 (2017);, Erratum: Phys.Rev.Lett. 121, 129901 (2018).

[5] B. P. Abbott et al. (LIGO Scientific and Virgo Collaborations), GW170814: A Three-Detector Observation of Gravitational Waves from a Binary Black Hole Coalescence, Phys. Rev. Lett. 119, 141101 (2017).

[6] B. P. Abbott et al. (LIGO Scientific and Virgo Collaborations), GW170817: Observation of Gravitational Waves from a Binary Neutron Star Inspiral, Phys. Rev. Lett. 119, 161101 (2017).

[7] B. . P. . Abbott et al. (LIGO Scientific and Virgo Collaborations), GW170608: Observation of a 19-solar-mass Binary Black Hole Coalescence, Astrophys. J. 851, L35 (2017).

[8] B. P. Abbott et al. (LIGO Scientific and Virgo Collaborations), GWTC-1: A Gravitational-Wave Transient Catalog of Compact Binary Mergers Observed by LIGO and Virgo during the First and Second Observing Runs, Phys. Rev. X 9, 031040 (2019).

[9] B. P. Abbott et al. (LIGO Scientific and Virgo Collaborations), GW190425: Observation of a compact binary coalescence with total mass $\sim 3.4 M_{\odot}$, Astrophys. J. Lett. 892, L3 (2020).
[10] R. Abbott et al. (LIGO Scientific and Virgo Collaborations), GW190412: Observation of a binary-black-hole coalescence with asymmetric masses, Phys. Rev. D 102 , 043015 (2020).

[11] R. Abbott et al. (LIGO Scientific and Virgo Collaborations), GW190814: Gravitational waves from the coalescence of a 23 solar mass black hole with a 2.6 solar mass compact object, Astrophys. J. Lett. 896, L44 (2020).

[12] R. Abbott et al. (LIGO Scientific and Virgo Collaborations), GW190521: A Binary Black Hole Merger with a Total Mass of $150 M_{\odot}$, Phys. Rev. Lett. 125, 101102 (2020).

[13] R. Abbott et al. (LIGO Scientific and Virgo Collaborations), GWTC-2: Compact Binary Coalescences Observed by LIGO and Virgo During the First Half of the Third Observing Run, arXiv:2010.14527 [Phys. Rev. X (to be published)].

[14] K. Danzmann, LISA: An ESA cornerstone mission for a gravitational wave observatory, Classical Quantum Gravity 14, 1399 (1997).

[15] P. Amaro-Seoane et al. (LISA Collaboration), Laser Interferometer Space Antenna, arXiv:1702.00786 [astroph.IM].

[16] J. Luo et al. (TianQin Collaboration), TianQin: a spaceborne gravitational wave detector, Classical Quantum Gravity 33, 035010 (2016).

[17] W.-R. Hu and Y.-L. Wu, The Taiji program in space for gravitational wave physics and the nature of gravity, Natl. Sci. Rev. 4, 685 (2017).

[18] B. F. Schutz, Determining the Hubble Constant from Gravitational Wave Observations, Nature (London) 323, 310 (1986).

[19] B. P. Abbott et al. (LIGO Scientific, Virgo, 1M2H, Dark Energy Camera GW-E, DES, DLT40, Las Cumbres Ob- 
servatory, VINROUGE, MASTER Collaborations), A gravitational-wave standard siren measurement of the Hubble constant, Nature (London) 551, 85 (2017).

[20] H.-Y. Chen, M. Fishbach, and D. E. Holz, A two per cent Hubble constant measurement from standard sirens within five years, Nature 562, 545 (2018).

[21] W. J. Wolf and M. Lagos, Standard Sirens as a Novel Probe of Dark Energy, Phys. Rev. Lett. 124, 061101 (2020).

[22] A. G. Riess, S. Casertano, W. Yuan, L. M. Macri, and D. Scolnic, Large Magellanic Cloud Cepheid Standards Provide a $1 \%$ Foundation for the Determination of the Hubble Constant and Stronger Evidence for Physics beyond $\Lambda$ CDM, Astrophys. J. 876, 85 (2019).

[23] S. Fairhurst, Triangulation of gravitational wave sources with a network of detectors, New J. Phys. 11, 123006 (2009); Erratum: New J.Phys. 13, 069602 (2011).

[24] S. Fairhurst, Source localization with an advanced gravitational wave detector network, Classical Quantum Gravity 28, 105021 (2011).

[25] K. Grover, S. Fairhurst, B. F. Farr, I. Mandel, C. Rodriguez, T. Sidery, and A. Vecchio, Comparison of Gravitational Wave Detector Network Sky Localization Approximations, Phys. Rev. D 89, 042004 (2014).

[26] W.-H. Ruan, C. Liu, Z.-K. Guo, Y.-L. Wu, and R.-G. Cai, The LISA-Taiji network: precision localization of massive black hole binaries, Research 2021, 6014164 (2021).

[27] W.-H. Ruan, C. Liu, Z.-K. Guo, Y.-L. Wu, and R.-G. Cai, The LISA-Taiji network, Nat.Astron. 4, 108 (2020).

[28] G. Wang, W.-T. Ni, W.-B. Han, S.-C. Yang, and X.Y. Zhong, Numerical simulation of sky localization for LISA-TAIJI joint observation, Phys. Rev. D 102, 024089 (2020).

[29] W.-F. Feng, H.-T. Wang, X.-C. Hu, Y.-M. Hu, and Y. Wang, Preliminary study on parameter estimation accuracy of supermassive black hole binary inspirals for TianQin, Phys. Rev. D 99, 123002 (2019).

[30] S.-J. Huang, Y.-M. Hu, V. Korol, P.-C. Li, Z.-C. Liang, Y. Lu, H.-T. Wang, S. Yu, and J. Mei, Science with the TianQin Observatory: Preliminary results on Galactic double white dwarf binaries, Phys. Rev. D 102, 063021 (2020).

[31] C. Cutler, Angular resolution of the LISA gravitational wave detector, Phys. Rev. D 57, 7089 (1998).

[32] A. Blaut, Accuracy of estimation of parameters with LISA, Phys. Rev. D 83, 083006 (2011).
[33] C. Zhang, Y. Gong, B. Wang, and C. Zhang, Accuracy of estimation of parameters with space-borne gravitational wave observatory, arXiv:2012.01043 [gr-qc].

[34] D. M. Eardley, D. L. Lee, and A. P. Lightman, Gravitational-wave observations as a tool for testing relativistic gravity, Phys. Rev. D 8, 3308 (1973).

[35] D. Liang, Y. Gong, S. Hou, and Y. Liu, Polarizations of gravitational waves in $f(R)$ gravity, Phys. Rev. D 95, 104034 (2017).

[36] S. Hou, Y. Gong, and Y. Liu, Polarizations of Gravitational Waves in Horndeski Theory, Eur. Phys. J. C 78, 378 (2018).

[37] Y. Gong and S. Hou, Gravitational wave polarizations in $f(R)$ gravity and scalar-tensor theory, EPJ Web Conf. 168, 01003 (2018).

[38] Y. Gong, E. Papantonopoulos, and Z. Yi, Constraints on scalar-tensor theory of gravity by the recent observational results on gravitational waves, Eur. Phys. J. C 78, 738 (2018).

[39] Y. Gong, S. Hou, D. Liang, and E. Papantonopoulos, Gravitational waves in Einstein-æther and generalized TeVeS theory after GW170817, Phys. Rev. D 97, 084040 (2018).

[40] Y. Gong and S. Hou, The polarizations of gravitational waves, Universe 4, 85 (2018).

[41] Y. Gong, S. Hou, E. Papantonopoulos, and D. Tzortzis, Gravitational waves and the polarizations in Hořava gravity after GW170817, Phys. Rev. D 98, 104017 (2018).

[42] S. Hou and Y. Gong, Gravitational waves in EinsteinÆther theory and generalized TeVeS theory after GW170817, Universe 4, 84 (2018).

[43] F. B. Estabrook and H. D. Wahlquist, Response of Doppler spacecraft tracking to gravitational radiation, Gen. Relat. Gravit. 6, 439 (1975).

[44] N. J. Cornish and S. L. Larson, Space missions to detect the cosmic gravitational wave background, Classical Quantum Gravity 18, 3473 (2001).

[45] X.-C. Hu, X.-H. Li, Y. Wang, W.-F. Feng, M.-Y. Zhou, Y.-M. Hu, S.-C. Hu, J.-W. Mei, and C.-G. Shao, Fundamentals of the orbit and response for TianQin, Classical Quantum Gravity 35, 095008 (2018).

[46] A. Vecchio and E. D. L. Wickham, The Effect of the LISA response function on observations of monochromatic sources, Phys. Rev. D 70, 082002 (2004).

[47] D. Liang, Y. Gong, A. J. Weinstein, C. Zhang, and C. Zhang, Frequency response of space-based interferometric gravitational-wave detectors, Phys. Rev. D 99, 104027 (2019). 